\title{
Study of combined THGEM/GAPD-matrix multiplier in a two-phase Cryogenic Avalanche Detector in Ar
}

\author{
A. Bondar ${ }^{1,2}$, A. Buzulutskov ${ }^{1,2}$, A. Dolgov ${ }^{2}$, E. Shemyakina ${ }^{1,2}$, and A. Sokolov ${ }^{1,2, a}$ \\ ${ }^{1}$ Budker Institute of Nuclear Physics SB RAS, Lavrentiev avenue 11, 630090 Novosibirsk, Russia \\ ${ }^{2}$ Novosibirsk State University, Pirogov street 2, 630090 Novosibirsk, Russia
}

\begin{abstract}
Two-phase Cryogenic Avalanche Detectors (CRADs) with combined THGEM/GAPD-matrix multiplier have become an emerging technique in rare-event experiments such as those of coherent neutrino-nucleus scattering and dark matter search. In this work we continue to study the performance of the two-phase CRAD in Ar with THGEM/GAPD-matrix charge/optical readout. The matrix was composed of a 3x3 array of GAPDs (Geiger-mode APDs), optically recording THGEM-hole avalanches in the Near Infrared (NIR). Gain, time, amplitude and spatial resolution properties of the combined multiplier are described.
\end{abstract}

\section{Introduction}

The development of two-phase Cryogenic Avalanche Detectors (CRADs) based on noble liquids [1] is relevant in the field of rare-event experiments for dark matter search [2] and coherent neutrino-nucleus scattering [3, 4]. In two-phase CRADs the ionization electrons produced in the noble liquid by a particle are extracted into the gas phase and multiplied with GEM or THGEM [5] multipliers. The multiplied electrons can be recorded directly using a charge readout $[6,7]$ or indirectly using an optical readout of avalanche photons with either Geiger-mode APDs (GAPDs) [8] or CCD camera [9]. Gain values as large as 1000 were obtained in a two-phase CRAD operated in Ar with the double-THGEM multiplier [10]. This gain value is however not enough for effective operation with ultimate sensitivity, i.e. in single-electron counting mode and with precise 2D readout.

A solution of this problem is to use a combined THGEM/GAPD-matrix multiplier, where the GAPDs record avalanche scintillations from the THGEM holes either in the Vacuum Ultraviolet (VUV) [8] (using wavelength shifters), Near Infrared (NIR) $[11,12]$ or Near U1traviolet [13] spectral range. In this work we continue to study the performance of the two-phase CRAD in Ar with THGEM/GAPD-matrix charge/optical readout; first results of the performance of such a combined multiplier were presented elsewhere [14].

\section{Experimental setup}

The experimental setup was identical to that used in our previous study of the two-phase CRAD in Ar with combined THGEM/GAPD-matrix multiplier [14]. It included

\footnotetext{
a e-mail: A.V.Sokolov@inp.nsk.su
}

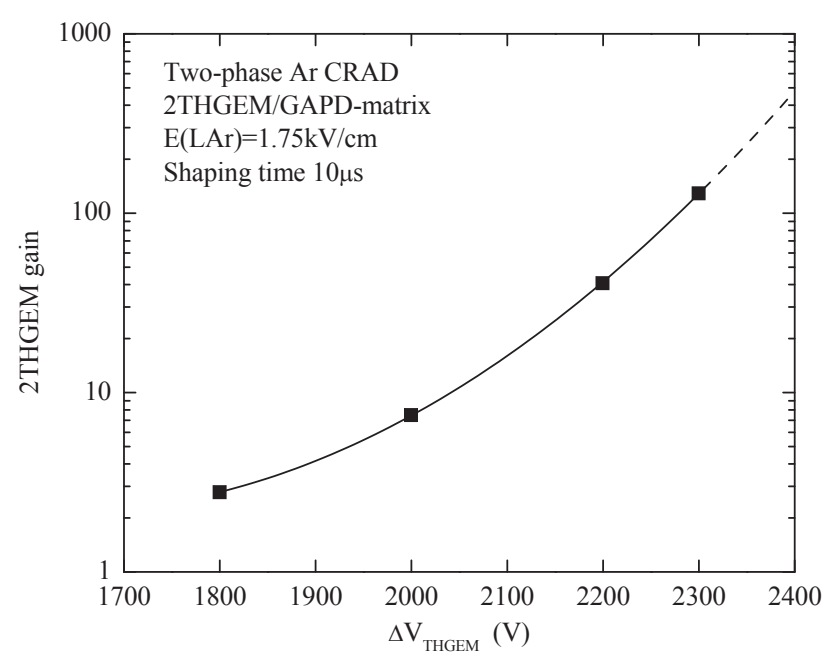

Figure 1. Charge gain of the double-THGEM multiplier as a function of the applied voltage on each THGEM, in a two-phase CRAD in Ar at $87 \mathrm{~K}$.

a cryostat with a 9 liters volume cryogenic chamber. The chamber consisted of a cathode mesh, immersed in a $\sim 1 \mathrm{~cm}$ thick liquid-Ar layer, and a double-THGEM assembly with an active area of $10 \times 10 \mathrm{~cm}^{2}$, placed in the gas phase above the liquid. A $3 \times 3$ matrix of GAPDs (MRS APD "CPTA 149-35") was placed in the gas phase at a distance of $6.5 \mathrm{~mm}$ behind the second THGEM, electrically insulated from the latter with an acrylic plate transparent in the NIR and by a wire grid at ground potential. Each GAPD had a $2.1 \times 2.1 \mathrm{~mm}^{2}$ active area and a Photon Detection Efficiency (PDE) of about $15 \%$ at $800 \mathrm{~nm}$. The detector was operated in two-phase mode in the equilibrium state, at a saturated vapor pressure of $1.0 \mathrm{~atm}$ and at a temperature of $87 \mathrm{~K}$. The Ar was purified by an Ox- 
isorb filter, providing an electron life-time of $>13 \mu \mathrm{s}$ in the liquid. In this study, the charge gain of the doubleTHGEM multiplier was kept at rather moderate level, in the range of 40-160 (see Fig. 1). The chamber has aluminum windows at the bottom for irradiation with pulsed X-rays. The detector was irradiated by $15-40 \mathrm{keV}$ X-rays from a pulsed X-ray tube with Mo anode operated at 40 $\mathrm{kV}$, with a pulse rate of $240 \mathrm{~Hz}$, through a cylindrical collimator with a hole diameter of $2 \mathrm{~mm}$. The X-ray conversion in the liquid was defined by this collimator and occurred over a small area of $2 \mathrm{~mm}$ in diameter. The charge signals were recorded from the last electrode of the second THGEM using a charge-sensitive amplifier. The DAQ system included an 8-channel Flash ADC CAEN V1720 (12 bits, $250 \mathrm{MHz}$ ): the signals from the THGEM were digitized and stored in a computer for further off-line analysis using a LabView ${ }^{\mathrm{TM}}$ program.

\section{GAPD signals}

Fig. 2 depicts a typical signal from the GAPD. The GAPD signals are composed of a number of fast pulses (peaks), each apparently corresponding to a single photoelectron detection followed by a single-pixel discharge (or those of multi-pixels in case of cross-talks).

As seen from the upper figure in Fig. 2, the signal width exceeds few microseconds. This is due to the potential barrier at the liquid-gas interface, that delays some of the electrons for a sufficiently long time, sometimes exceeding $20 \mu \mathrm{s}$.

This is well illustrated in Fig. 3, where one can clearly see the fast and slow components of the signal. The fast component corresponds to the signal from the electrons, which directly penetrate through the potential barrier; the slow component corresponds to the electrons that were delayed by a potential barrier. The specific shape of the leading edge of the fast component in the distribution of Fig. 3 is due to the presence of harder and softer components in the spectrum of X-rays absorbed in the liquid Ar layer. Softer and harder X-rays are absorbed at different depths, and have, accordingly, different electron drift times. For details of the spectrum shape of pulsed X-rays absorbed in liquid $\mathrm{Ar}$ and their average deposited energy, amounting to $25 \mathrm{keV}$, see ref. [15].

In Fig. 2 one can see that the signals from the GAPD have rather different pulse-heights, with the tendency to smaller values. This is due to the effect of the GAPD performance degradation at lower temperatures observed recently $[16,17]$. This effect manifested itself at higher photon fluxes; it resulted from the increase of the GAPD pixel recovery time due to the increase of the pixel quenching resistor at cryogenic temperatures. Some pulses were even lost below threshold, thus reducing the photon detection efficiency of the GAPD. Our recent studies [17] showed that this problem can be solved using a different type of GAPD, namely those of the MPPC type by Hamamatsu production, with reduced resistance of the pixel quenching resistor.

Since in the current measurements the GAPD pulseheight was degraded as shown above, the GAPD ampli-
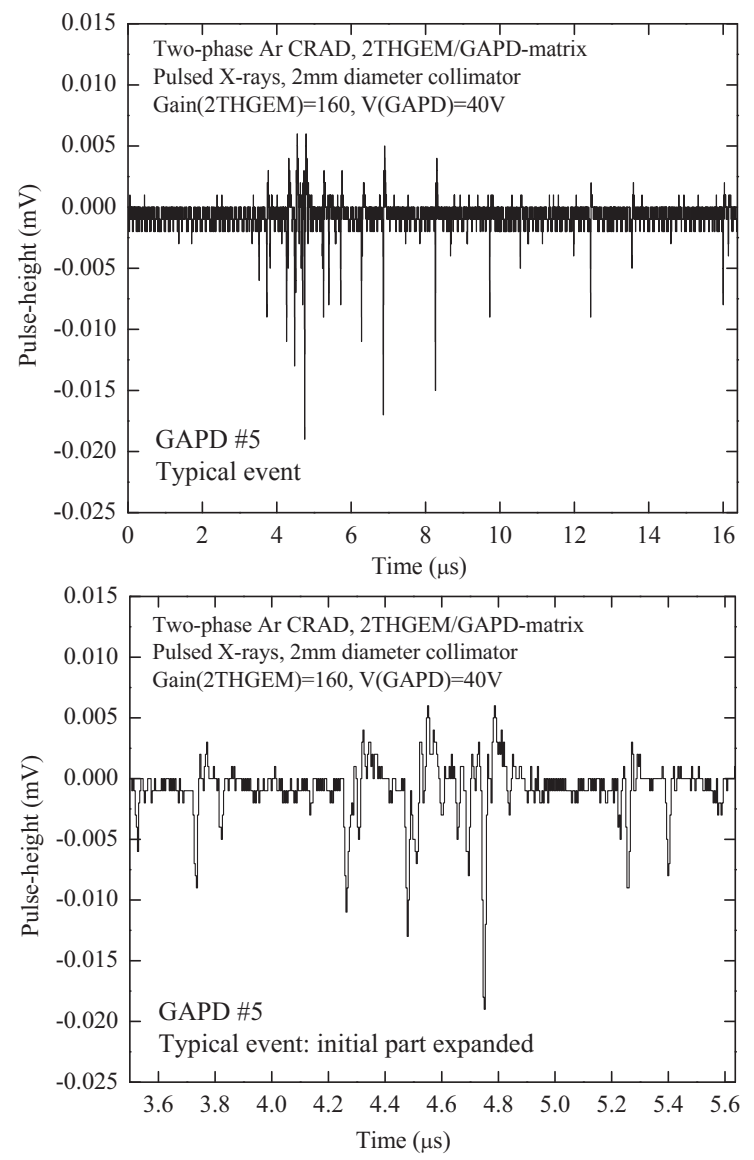

Figure 2. In the upper figure the typical signal of the GAPD in the two-phase CRAD in Ar with a THGEM/GAPD-matrix multiplier is shown. The lower figure represents the initial part of the GAPD signal. As one can see the signals have different amplitudes due to the degradation effect.

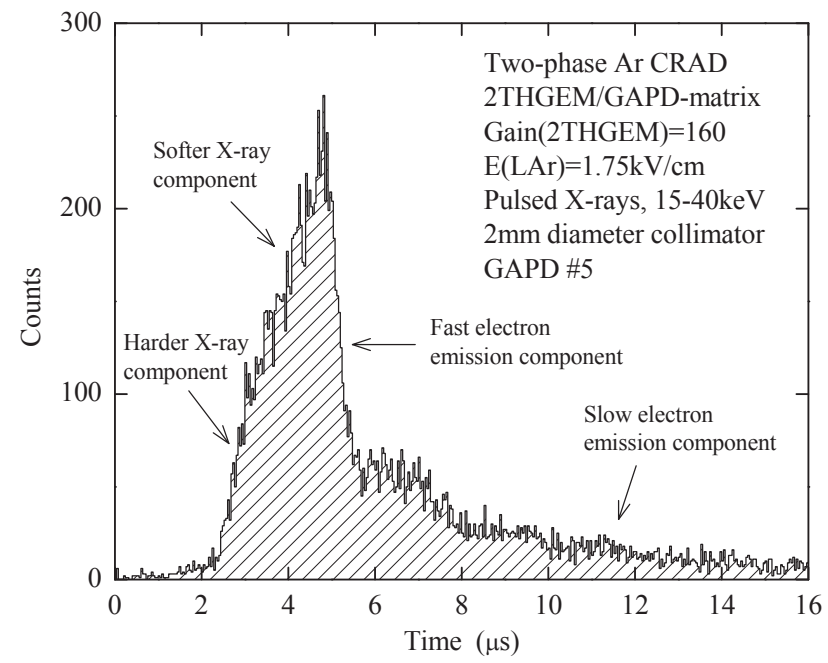

Figure 3. Time distribution of the GAPD signals with respect to the X-ray pulse trigger, in the two-phase CRAD in Ar with THGEM/GAPD-matrix multiplier, induced by pulsed X-rays. The figure clearly shows the fast and slow components of the signals. 


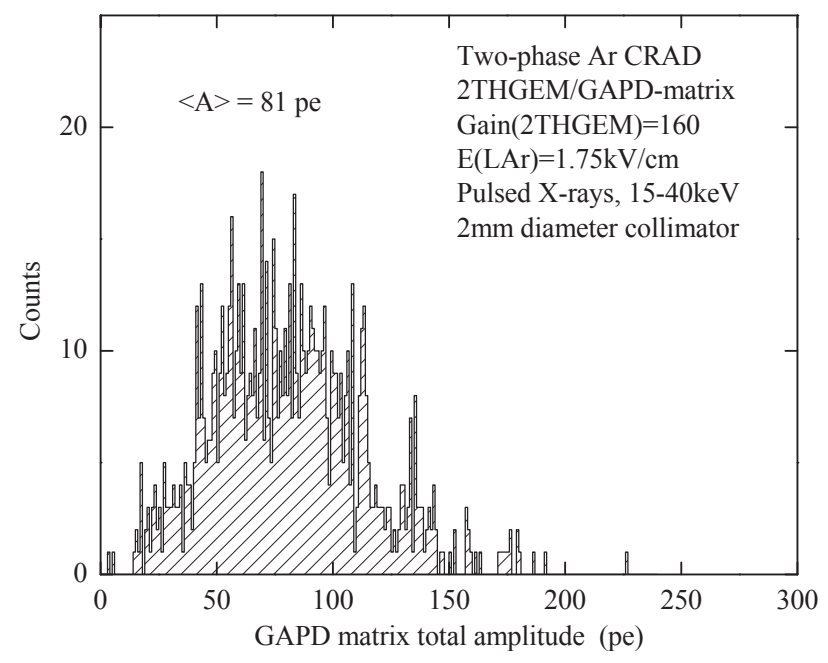

Figure 4. Distribution of GAPD-matrix total amplitude expressed in the number of photoelectrons detected, in a two-phase CRAD in Ar with THGEM/GAPD-matrix multiplier, induced by pulsed X-rays absorbed in liquid Ar with the average energy of $25 \mathrm{keV}$.

tude was calculated by just counting the number of photoelectron peaks in the signal. The result for the total GAPD-matrix amplitude is shown in Fig. 4. One can see that yield of the combined THGEM/GAPD-matrix multiplier in present conditions, namely at the double-THGEM charge gain of 160 , was high enough, amounting to 81 photoelectrons per $25 \mathrm{keV}$ deposited energy corresponding to the reduced multiplier yield of about 3 photoelectrons per $\mathrm{keV}$.

\section{Coordinate characteristics}

The X-Y coordinate characteristics of the THGEM/GAPD-matrix multiplier are illustrated in Figs. 5 and 6. The X-Y coordinates were calculated from the GAPDs amplitudes using a center-of-gravity algorithm, corrected for Monte-Carlo simulation of the light propagation from the THGEM holes to a given GAPD. The result of such simulation is shown in Fig. 5. The curve in the figure, obtained by fitting to the simulated data points, was used to reconstruct the true coordinate from that of calculated using the center-of-gravity algorithm.

Fig. 6 illustrates the imaging capability of the detector: the X-Y coordinate plot shows the pulsed X-ray conversion regions defined by the $2 \mathrm{~mm}$ diameter collimator.

Fig. 7 shows the Y projection of the coordinate distribution. The width of distribution is $3.2 \mathrm{~mm}$ (FWHM). The spatial resolution of the detector can be estimated from the data by quadratic subtraction of the collimator hole diameter; it results in the value of $1 \mathrm{~mm}$ (a standard deviation of the distribution) for $25 \mathrm{keV}$ energy deposition, at the present operation conditions.

\section{Conclusions}

A multi-channel optical readout in a two-phase Cryogenic Avalanche Detector (CRAD) in Ar with combined

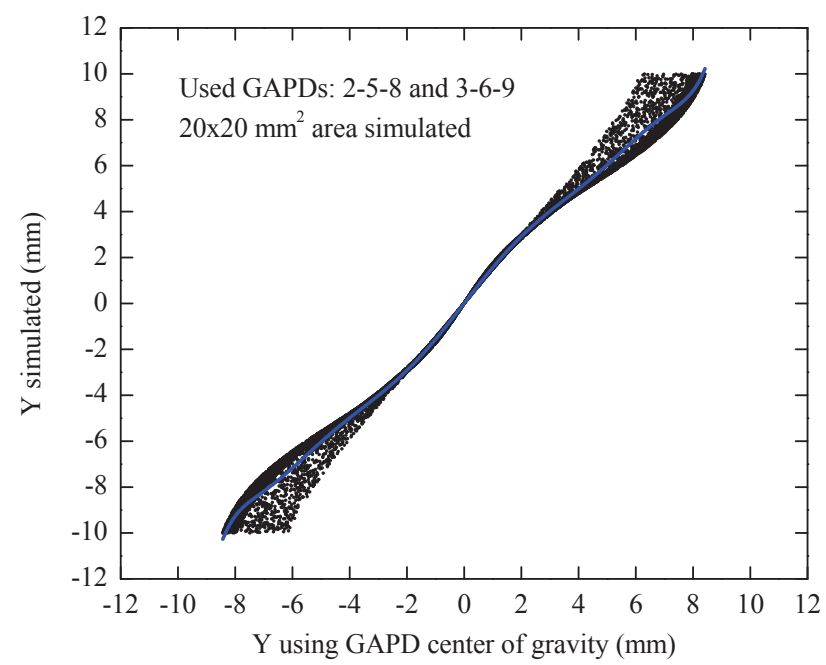

Figure 5. Simulated Y-coordinate versus that of reconstructed using 2-5-8 and 3-6-9 GAPD columns of the GAPD matrix of Fig. 6). The blue curve is the fit to the data points.

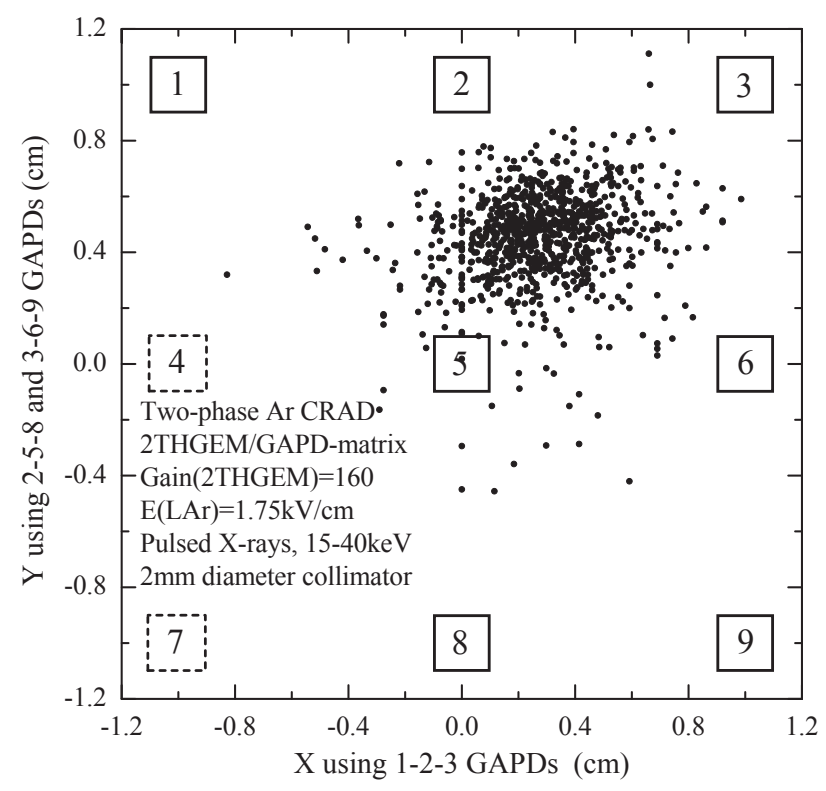

Figure 6. $\mathrm{X}-\mathrm{Y}$ plots of the pulsed $\mathrm{X}$-ray conversion region in the two-phase CRAD in Ar with THGEM/GAPD-matrix multiplier, defined by a $2 \mathrm{~mm}$ diameter collimator. Squares with numbers indicate the positioning of the active areas of the appropriate GAPDs of the matrix.

THGEM/GAPD-matrix multiplier has been studied. The detector had a high yield of combined multiplier, of 3 photoelectrons per keV of deposited energy, and a good spatial resolution $\sigma=1 \mathrm{~mm}$ for $25 \mathrm{keV}$ X-rays. Such a readout can be applied in low threshold rare-event experiments like those of coherent neutrino-nucleus scattering and dark matter searches. 


\section{Acknowledgements}

We thank A. Chegodaev and R. Snopkov for technical support. This work was supported by the Russian Science Foundation (project N 14-50-00080).

\section{References}

[1] Buzulutskov A., JINST 7, C02025, 2012.

[2] Akimov D. et al., NIM vol 598 P 275, 2009.

[3] Hagmann C., Bernstein A., IEEE Trans. Nucl. Sci. vol 51 P 2151 (2004).

[4] Akimov D. et al., JINST 4, P06010, 2009.

[5] A. Breskin et al., Nucl. Instrum. Meth. A 598 (2009) 107.

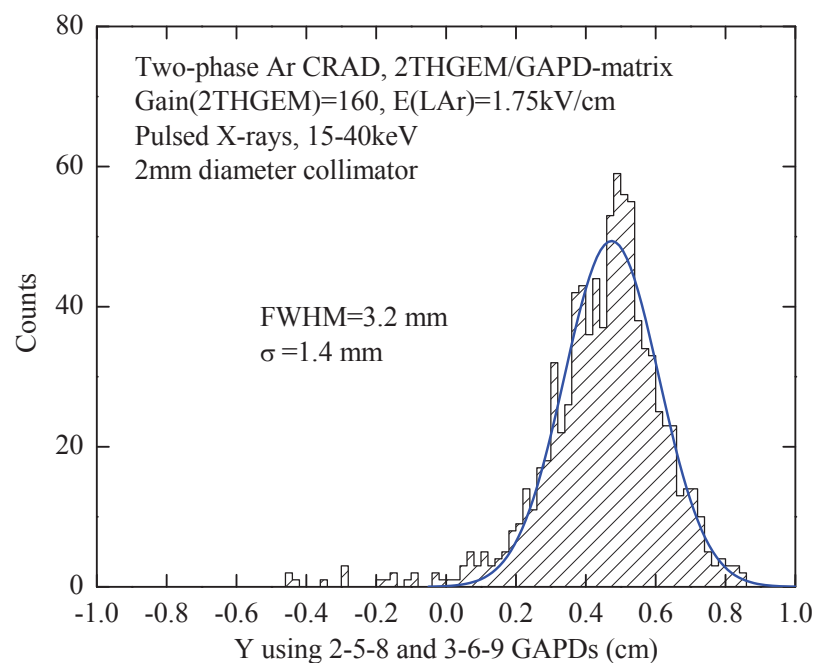

Figure 7. $\mathrm{Y}$ projection of 2D-coordinate distribution of the pulsed X-ray conversion region in the two-phase Ar CRAD with THGEM/GAPD-matrix optical readout, defined by $2 \mathrm{~mm}$ diameter collimator when irradiated with pulsed $\mathrm{X}$-rays.
[6] A. Bondar et al., JINST 8, P02008, 2013.

[7] A. Badertscher et al., Nucl. Instrum. Meth. A 641 (2011) 48.

[8] P.K. Lightfoot et al., JINST 4, P04002, 2009.

[9] K. Mavrokoridis et al., JINST 10, P10004, 2015.

[10] A. Bondar et al., JINST 8, P02008, 2013.

[11] A. Bondar et al., JINST 5, P08002, 2010.

[12] A. Bondar et al., JINST 7, P06014, 2012.

[13] A. Bondar et al., EPL 112, 19001, 2015.

[14] A. Bondar et al., Nucl. Instrum. Meth. A 732 (2013) 213.

[15] A. Bondar et al., Eprint arXiv:1505.02296, 2015.

[16] A. Bondar et al., JINST 9, P08006, 2014.

[17] A. Bondar et al., JINST 10, P04013, 2015. 